\title{
Maternal and paternal psychopathological risk in children with Non Organic Failure to Thrive
}

Recent researches have underlined that maternal psychopathology can be considered as a crucial risk factor for the development and maintaining of several emotional behavioural problems in children. While several researches have dealt prominently with maternal psychopathological risk and with their outcomes on NOFTT, limited attention has been given in literature to fathers' involvement in caregiving and to their psychological profiles, while this issue may represent a significant risk and/or protective factor. The present longitudinal study is aimed to further elaborate on the impact of the maternal psychological functioning on the adaptation of the child between the first and second childhood, focusing also on the paternal involvement in caregiving. 


\title{
Maternal and paternal psychopathological risk in children with non-organic failure to thrive
}

\author{
Cerniglia Luca ${ }^{1}$, Cimino Silvia ${ }^{2}$ \\ ${ }^{1}$ International Telematic University Uninettuno, Rome, Italy \\ ${ }^{2}$ Sapienza University of Rome, Italy
}

\begin{abstract}
Introduction and aims

Recent researches have underlined that maternal psychopathology can be considered as a crucial risk factor for the development and maintaining of several emotional behavioural problems in children (Cimino, Cerniglia, Paciello, Sinesi, 2012; Cox e Barton, 2010).

Several researches found evidence that maternal psychological characteristics can affect infant weight gain or predispose children to weight faltering. Specifically, robust associations have been found between maternal depression and Organic and Non Organic Failure to Thrive in infancy (Wright, Parkinson \& Drewett, 2006).

It has also been underlined that emotional difficulties in mothers can obstacle their caregiving skills and the capacity of acting as regulatory mediators of problematic internal states of their children (Cerniglia, Cimino, Ballarotto, Monniello, 2014). Furthermore, it has been observed that mothers who show symptoms of emotional distress, anxiety, somatization, and difficulty with their own regulation have strong probabilities to have children with difficulties in modulating their own emotions and their impulses (Bryant-Waugh, Markham, Kreipe \& Walsh, 2010; Cimino, Cerniglia,
\end{abstract} Paciello, 2015).

Recent studies have enlightened that depressive symptoms in mothers can have an impact on emotional quality of parenting. Mothers who suffer from depression seem to provide less quantity of food to their children, which may be associated to weight faltering.

Olsen and co-workers (Olsen, Petersen, Skovgaard, Weile, Jørgensen, \& Wrighth, 2007) have suggested that a single paediatric assessment may not be adequate to identify nutritional growth delay in the general population making longitudinal population studies more adequate to explore the contribution of different criteria for NOFTT and to predict outcomes such as neurodevelopmental and behavioural problems.

While several researches have dealt prominently with maternal psychopathological risk and with their outcomes on NOFTT, limited attention has been given in literature to fathers' involvement in 
caregiving and to their psychological profiles, while this issue may represent a significant risk and/or protective factor.

It has been emphasized that an appropriate paternal psychological functioning allows a better comprehension of children's needs and represents a protective factor versus possible psychopathological outcomes (Cooper, Whelan, Woolgar, Morrell, Murray, 2004; Reif et al., 1995).

The scientific literature has relatively deepened the interconnection between maternal and paternal characteristics and weight faltering in their children.

Moreover, the presence of psychopathologic symptoms in partners of women with severe eating disorders precludes adequate mother-infant interactions during feeding and facilitates the onset of emotional disorders in the children (Dietz, Jennings, Kelley, Marshal, 2009).

These researches underline the importance of deepening the role of the fathers' involvement both in samples of families without NOFTT and in families where children are manifesting early emotional-adaptive difficulties, considering the evolution of the psychological and/or psychopathological profile of parents and children in the course of time.

In fact, the scientific literature that has studied the outcomes of maternal emotional difficulties (e.g. maternal eating disorders) on psychological functioning of the child has not taken into appropriate consideration the role of the father as potential risk or protective factor.

Basing on these theoretical foundations, the present longitudinal study is aimed to further elaborate on the impact of the maternal psychological functioning on the adaptation of the child between the first and second childhood, focusing also on the paternal involvement in caregiving.

Specific objectives are as follows:

1) Evaluating the trend of psychological profile of the mothers and fathers in three different assessment sessions;

2) Evaluating the child malnutrition and emotional/adaptive profile described by mothers and fathers in three different assessment sessions;

3) Evaluating the impact of the specific maternal and paternal profile risks related to child malnutrition and to the presence of maladaptive pattern among emotional/adaptive functioning variables defined by both mothers and fathers in three different assessment sessions.

\section{Method}

Sample

The sample is composed of $\mathrm{N}=60$ parents with children that have followed a longitudinal research protocol and have been recruited through nurseries, primary schools and outpatients' clinics. It was part of a larger study included in a research agreement between the Faculty of Psychology, Sapienza University of Rome and paediatric hospitals in Rome and in Central Italy. 
The longitudinal assessment were completed in 6 years, when children were 2 (Time 1), 5 (Time 2) and 8 years old (Time 3 ).

At first assessment session (T1) N=297 couples of parents have been recruited. Consistently with several prospect studies (Kotler et al., 200; Timimi et al., 1997), the mortality sample rate at the second (T2) and third evaluation time (T3) was respectively about 53\% and 74\%. Most of the couples of parents were married (93\%) and had obtained a secondary school diploma (82\%). The gestational age and psychomotor development of all the children, at the time of the first assessment, were in the normal range. Most of the children had been breastfed (81\%) and were firstborn (79\%). Most parents (87\%) were of middle socio-economic status (SES- Hollingsead, 1975).

On the basis of a nutritional evaluation done by paediatrician in T1 (following Waterloo criteria), the sample has been split into two groups: couples of parents with children affected by Non Organic Failure to Thrive (CG, $\mathrm{N}=15)$ and couples of parents of children with growth consistent with their age, which are here defined as non clinical group ( $\mathrm{NCG}, \mathrm{N}=15$ ). The two groups have been paired by age of the children, age of the mothers and families' socio-economic status.

The children's mean age in the CG was 2.6 (years, months) at Time 1 (S.D. 0.7), 5.3 (S.D. 0.5) at Time 2 and 7.6 (S.D. 0.4) at Time 3. The children's mean age in the NCG was 2.2 (years, months) at Time 1 (S.D. 0.2), 5.1 (S.D. 0.6) at Time 2 and 7.7 (S.D. 0.5) at Time 3. At Time 1, maternal mean age for GC was 31.4 and 32 years for NCG (S.D. 3.6 and 4.6, respectively).

A set of self-report and report-form tools, along with children nutritional evaluation made by the paediatrician, has been administered to both parents in a longitudinal protocol and all parents signed an informed consent for all the procedures.

\section{Tools and procedures}

At three evaluation times (T1, T2, T3) both Clinical Group (CG) and Non Clinical Group (NCG) have been assessed through:

SCID-I: Structured Clinical Interview for DSM-IV Axis I Disorders (SCID-I), a semi-structured interview addressing the major DSM-IV Axis I diagnoses (First, Spitzer, Gibbon, Williams, 1997). The SCID-I is divided into six self-contained modules that can be administered in sequence: $\operatorname{mood}$ episodes; psychotic symptoms; psychotic disorders; mood disorders; substance use disorders; anxiety, adjustment, and other disorders. This tool has been administered only at Time 1.

SCL-90-R: The Symptom Checklist-90-Revised (SCL-90-R), a 90-item self-report symptom inventory aimed to measure psychological symptoms and psychological distress. The principal symptom dimensions are: Somatization, Obsessive-Compulsive, Interpersonal Sensitivity, Depression, Anxiety, Hostility, Phobic Anxiety, Paranoid Ideation and Psychoticism. The internal 
coherence tested within a sample of adolescent and adults is satisfying (alpha between 0.70 and 0.96) and clinical cut-off $=1$ (Prunas et al., 2011).

CBCL: The Child Behavior Checklist (CBCL), a questionnaire filled out by parents and caregivers to assess the child's abilities and specific behavioral/emotional attributes. The CBCL/1 1/2 - 5 (Achenbach \& Rescorla, 2001) is composed of 100 items. The Internalizing Problem scale consists of the Emotionally Reactive, Anxious/Depressed, Somatic Complaints and Withdrawn subscales. The Externalizing Problem scale consists of the Attention Problems and Aggressive Behavior subscales. The $C B C L / 1 \frac{1 / 2-5}{2}$ has high test-retest reliability, high internal consistency (Achenbach \& Rescorla, 2001). The CBCL/6-18 (Achenbach \& Rescorla, 2001) is composed of 113 items. The Internalizing Problem scale consists of Anxious/Depressed, Withdrawn/Depressed and Somatic Complaints; the Externalizing Problem scale consists of Rule-Breaking Behavior and Aggressive Behavior. Both in CBCL/6-18 and CBCL/1 1/2 - 5 the internal broadband scores are valid and it has been observed that the scale is able to discriminate children affected by disorders from nonreferred children (D’Orlando et al., 2010).

\section{Results}

Evaluating the trend of psychological profile of the mothers and fathers in three different assessment sessions

According to $S C I D-I$ results, within $C G$, a sub-sample of $\mathrm{N}=16$ mothers have obtained a diagnosis for anorexia nervosa, and a sub-group of $\mathrm{N}=9$ mothers have been diagnosed for bulimia nervosa. On the other hand, within NCG none of the mothers have been diagnosed according to any SCID-I criteria. With regards to fathers, in $\mathrm{CG}$ a sample of $\mathrm{N}=6$ fulfilled the criteria for nonspecific psychological anxiety diagnosis; within NCG only one father $(\mathrm{N}=1)$ has fulfilled the diagnosis for a dysthymia.

The trend for $S C L-90-R$ scores at the three times of evaluation, showed no significant differences among maternal and paternal psychological profiles. Besides, their profiles have shown to be stable over the three assessment sessions. A significant effect of $S C L-90-R$ scores X Group interaction $(\mathrm{F}(4,224)=4.576 ; \mathrm{p}=0.001)$ have been found. The post-hoc comparisons have been highlighted that CG shows higher scores in all the dimensions of $S C L-90-R$ at T1, T2 and T3 in comparison with the $\mathrm{NCG}(\mathrm{p}<0.05)$.

In both samples significant differences have been identified between mothers and fathers' scores in the following dimensions: somatization $(\mathrm{F}(1,56)=16.459 ; \mathrm{p}<0.001)$, depression $(\mathrm{F}(1,56)=36.964$; $\mathrm{p}<0.001)$, obsessive-compulsive $(\mathrm{F}(1,56)=27.042 ; \mathrm{p}<0.001)$ and anxiety $(\mathrm{F}(1,56)=14.866$; $\mathrm{p}<0.001)$. Post-hoc comparisons have outlined higher scores of the mothers in the subscales somatization and depression, while fathers have shown higher values in the dimensions of anxiety. 
In general, CG scored significantly higher than NCG in the above dimensions $(p<0.05)$ exceeding the clinic cut-off over the three evaluation sessions.

Mothers and fathers have shown stable scores over the three assessment sessions. A significant effect of the Group X EAT-40 scores interaction has been found $(\mathrm{F}(4,224)=4.576$; $\mathrm{p}=0.001)$. Posthoc comparisons have highlighted that CG showed significantly higher scores on all the EAT-40 dimension over the three times of evaluation compared with GNC $(p<0.05)$.

In CG, but not in NCG, a statistical difference has been found between mothers' and fathers' scores at EAT-40 $(1,56)=146.332 ; \mathrm{p}<0.001)$. Post-hoc analysis showed that the mothers' sample scored significantly higher values in the three examined subscales $(\mathrm{p}<0.001)$ exceeding the clinical cut-off over the three evaluation sessions.

Evaluating the child's malnutrition and emotional/adaptive profile described by mothers and fathers in three different assessment sessions:

With regards to Malnutrition of the children, a statistical difference between the two samples (CG and NCG) has been identified; no child in NGC showed a malnutrition status over the three evaluation sessions. With the aim of evaluating the potential change in the malnutrition level a non parametric analysis within CG has been executed. The Kendall test using repeated measures has highlighted a significant difference over the three evaluation sessions per $\left(\operatorname{chi}^{2}=51,51 ; \mathrm{p}<0,001\right)$. The medium ranks suggest a decrease of malnutrition between the first and the second evaluation session (medium ranks between 2,85 and 2,02) and a further decrease in the third assessment session (medium rank $=1,13$ ). Furthermore, as confirmation of the partial improvement of the malnutrition level, none of the children showed a severe level of malnutrition during the third evaluation session.

A statistical interaction have been identified between time factor and emotional-adaptive child profile evaluated with CBCL and the group $(\mathrm{F}(8,448)=15.374 ; \mathrm{p}<0.001)$. The post-hoc comparisons have highlighted that in CG and NCG, there is a significant decrease of the scores in all the CBCL subscales in the third evaluation session $(p<0.05)$. This trend is not confirmed in the anxious/depressed behaviour and withdrawn dimensions of the CG. There is the tendency to show high scores exceeding the clinical cut-off in the third session of evaluation. Furthermore the children belonging to CG show significantly higher scores than NCG in all the CBCL dimensions $(p<0.05)$ over the three session of evaluation.

Differences between mothers' and fathers' sample: both of the samples have been highlighted a significant difference for the scores regarding the emotional-adaptive functioning within the dimension linked to somatic complaints $(\mathrm{F}(1,56)=14.491 ; \mathrm{p}<0.001)$ and the area related to aggressive behaviour $(\mathrm{F}(81,56)=30.547 ; \mathrm{p}<0.001)$. The post-hoc comparisons identified higher scores among mothers on somatic complaints of the child and higher scores in fathers' sample in the 
aggressive behaviour. Moreover, in CG higher scores above cut-off on the above dimensions have been found $(\mathrm{p}<0.05)$ over the three observation sessions.

Evaluating the impact of the specific maternal and paternal profile risks related to child malnutrition and to the presence of maladaptive pattern among emotional/adaptive functioning variables defined by both mothers and fathers in three different assessment sessions:

Correlation analysis among maternal and paternal psychopathological variables, malnutrition and emotional-behavioural child profile have been executed. High and statistically significant correlations have been outlined among several of the dimensions investigated by parents' reportform tools. Starting from the correlations, it has been chosen to undertake a multiple regression analysis to evaluate the weight of the specific variables of risks (both maternal and paternal) on malnutrition of the children and on their emotional-adaptive profile. It resulted that paternal obsessive-compulsive dimension $\left(\mathrm{p}<.05 ; \mathrm{R}^{2}=.075\right)$ associated with maternal depressive risk $(\mathrm{p}<.01$, $\left.\mathrm{R}^{2}=.081\right)$ are significant predictive indicators of the child malnutrition over the three evaluation sessions. Those dimensions, considered per se, do not reach such significant levels to be considered predictive indicators of child malnutrition. Furthermore the EAT-40 maternal overall score can be considered a significant child malnutrition predictor in the first evaluation session $\left(\mathrm{p}<.05 ; \mathrm{R}^{2}=.078\right)$ and in the CBCL dimension of withdrawn/depressed evaluated by the mothers over the three evaluation sessions $\left(\mathrm{p}<.05, \mathrm{R}^{2}=.010\right)$. Somatization has proved to be a significant predictor $(\mathrm{p}<.05$, $\left.\mathrm{R}^{2}=.010\right)$ of the CBCL anxious/depressed behaviour variable evaluated by mothers over the three evaluation sessions.

\section{Discussion}

The results of the present study are consistent with the international scientific literature and offer some new interesting hints.

Our research has confirmed that children with severe difficulties in the area of feeding tend to have mothers who show problems in the same field (Cooper et al., 2004). The recent bulk of researches has mainly underlined the strong association between eating disorders in the mothers and feeding disorders in their children (Micali, Simonoff, Stahl, Treasure, 2011).

Our results confirm many researches that have shown that eating disorders in mothers may predict infants' poor physical growth (Ammaniti et al., 2011; Cooper et al., 2004; Gueron-Sela, Atzaba-Poria, Meiri, Yerushalmi, 2011). It might be suggested that, since these women are not able to recognize their own emotional needs from physical signals of hunger and satiety, they burden the interactions with their children during meals with projections and distorted interpretations that do not allow an empathic understanding of the requests manifested by the infants (Blisset et al., 2011; Carano et al., 2011; Cerniglia, Cimino, Ballarotto, 2014; Coulthard et al., 2004; Micali et al., 2009). 
The contributes that had associated NOFTT in children in the first years of life and maternal eating disorders and are still scarce (Whelan e Cooper, 2000). It has been suggested that individual characteristics of the child, such individual psychological profile and also difficulty in sucking, chewing, or swallowing can predict NOFTT but the role of maternal handling of the feeding dynamic and the quality of interaction with their children and the function of psychopathological risk of the mothers have also been underlined (Wright, Parkinson \& Drewett, 2006). Our results are coherent with Watkins and co-workers' studies (2011) in that children with NOFTT have a high probability to have a mother showing eating disorders during her life time.

The longitudinal configuration of our study allows to confirm that maternal psychopathological risk is linked to NOFTT in early childhood and it can be considered as a clinical occurrence frequently associated with maladaptive emotional and psychological functioning in later phases of infancy (Bagner, Pettit, Lewinsohn, Seeley, 2010; Watkins, Cooper, Lask, 2011). In fact, the diagnosis of eating disorder in the mothers seems to predict failure to thrive in the children only at Time 1, while it is associated with a maladaptive psychological emotional functioning both in the first and in the second childhood. In this sense, it can be argued that NOFTT is a risk factor for the subsequent onset of emotional and affective dysregulation in childhood. We hypotize that maternal psychopathological risk influences the interactions with the children bringing vulnerability for NOFTT in the first three years of life and progressively affects more systemic issues of emotional functioning which remain problematic in Time 2 and 3, while malnutrition decreases.

Children with NOFTT seem also to be more likely to have parents with problematically stable psychological profiles. Both mothers and fathers of these children, in fact, show high values in several dimensions of psychopathological risk, such as somatization and depression (mothers) and obsessive/compulsive behaviours and anxiety (fathers). In particular, maternal depressive risk has widely been considered as strongly related to disadaptive emotional functioning, scarce intake of food and feeding-related problems in infancy (Wright, Parkinson, Drewett, 2006). In the case of children with NOFTT, as in our sample, this assumption seems to not be completely verified. If, in fact, it is confirmed that maternal depressive symptoms play an important role in establishing and maintaining of malnutrition during childhood, we found that they can be considered as valid predictors of failure to thrive only in the association of paternal psychopathological risk (obsessive/compulsive behaviours).

These results are coherent with the work of Elgar and co-workers (2007) and seem to suggest that the paternal psychopathology might play a crucial role in the quality of caregiving modulating and integrating with the interactional mother-infant patterns; in particular, a father with psychopathologic symptoms that shows a low level of responsiveness to a child may represents a risk factor for the onset of child's maladaptive behaviour. The presence of a maternal 
psychopathology associated to a paternal dysfunctional psychological profile seems to create a coparenting dominated by negative interactive cycles with the children (Teubert, Pinquart, 2010).

Besides, the emotional difficulties of the child with NOFTT seem to be differently represented by mothers and fathers. The first, mainly refer of somatic symptoms in their child, whereas the latter primarily describe aggressive behaviours. These results might be related to the respective problematic areas of the parents that as for the mothers account specifically for distressing internal states, and for the fathers are more connected to externalizing issues (Cooper, Whelan, Woolgar, Morrell, Murray, 2004).

These results suggest to pay a closer attention to the role of paternal involvement and psychopathological risk in the policies of assessing and treating NOFTT in infancy.

\section{References}

Cerniglia L., Cimino S., Ballarotto G., (2014), Mother-child and Father-child interaction with their 24-month-old children during feeding: a study considering paternal involvement and the child's temperament in a community sample. Infant Mental Health Journal, 35, 5, 473-481. DOI: 10.1002/imhj.21466.

Cimino S., Cerniglia L., Paciello M. (2015), Mothers with depression, anxiety or eating disorders and longitudinal outcomes on their children: a study on paternal psychopathological risk. Child Psychiatry and Human Development, 46, 228-236 DOI: 10.1007/s10578-014-0462-6.

Cerniglia L., Cimino S., Ballarotto G., Monniello G. (2014), Parental loss during childhood and outcomes on adolescents' psychological profiles: a longitudinal study. Current Psychology, 33(4), 545-556. DOI: 10.1007/s12144-014-9228-3.

Cimino S, Cerniglia L., Paciello M., Sinesi S. (2012), A six-year prospective study on children of mothers with eating disorders: the role of paternal psychological profiles. European Journal of Eating Disorders Review, 21, 3, 238-246. DOI: 10.1002/erv.2218. 
Lucarelli L, Cimino S, D'Olimpio F, Ammaniti M (2013) Feeding disorders of early childhood: an empirical study of diagnostic subtypes. International Journal of Eating Disorders 46(2), 147-155. doi: 10.1002/eat.22057. 